\section{Therapieergebnisse bei der pulmonalen Tuberkulose: Outcome-Monitoring im nördlichen Niedersachsen}

Zusammenfassung: Fragestellung: In der Bundesrepublik Deutschland erfolgt keine standardisierte Dokumentation der Therapieergebnisse bei der Behandlung der Tuberkulose. Methodik: Wir untersuchten retrospektiv das dokumentierte Behandlungsergebnis bei 494 Patienten mit einer gesicherten Lungentuberkulose unter Anwendung der international empfohlenen Kriterien. Ergebnisse: Die mediane Nachbeobachtungsdauer betrug 62 Monate (36-180 Monate). Eine erfolgreiche Therapie konnte bei 378 (76,1\%) der Patienten dokumentiert werden. Nahezu ausnahmslos handelte es sich dabei um Heilungen mit kulturell bestätigtem Therapieerfolg $(n=375)$. Unter den 119 Patienten (23,9\%) ohne dokumentierten Therapieerfolg war ein Ausscheiden der Patienten aus der retrospektiven Auswertung bedingt durch keine weitere Dokumentation des Behandlungsergebnisses nach der stationären Betreuung die bei weitem häufigste Ursache ( $n=60 ; 51,2 \%)$, gefolgt vom Tod $(n=24 ; 19,8 \%)$, einer abgebrochenen Therapie $(n=22$; $18,3 \%$ ) und einem Therapieversagen ( $n=13 ; 10,7 \%)$. Bei den $\mathrm{Er}$ krankungscharakteristika zeigte sich, dass der Behandlungserfolg bei Patienten mit einem Tuberkuloserezidiv schlechter war als bei einer Ersterkrankung $(p=0,0003)$. Auch eine nachgewiesene Resistenz des Erregers gegen mindestens ein Standardmedikament führte häufiger zu einer nicht erfolgreichen Therapie $(p=0,04)$. Von besonderer Bedeutung für das Therapieergebnis war der Unterschied, ob die Patienten sowohl in der initialen Therapiephase als auch in der Stabilisierungsphase eine Standardtherapie unter Einschluss von Isoniazid und Rifampicin erhielten oder eine anders kombinierte Therapie eingesetzt wurde ( $p=0,039$ bzw. 0,0021). Schlussfolgerung: Unsere Untersuchung zeigt, dass bei der retrospektiven Erfassung des Therapieergebnisses deutlich schlechtere Ergebnisse gewonnen werden, als theoretisch erwartbar wäre. Eine Überprüfung dieser Situation durch die prospektive Anwendung der internationalen Definitionen zum Monitoring des Therapieerfolgs bei der Tuberkulose erscheint daher notwendig.

Abstract: Background: Despite it importance standardized treatment outcome-monitoring in tuberculosis patients is not officially done in Germany. Methods: In this retrospective study we investigated tuberculosis outcome in 494 patients with pulmonary tuberculosis using the international recommended definitions. Results: The median follow-up period was 62 month (36-180 month). A successful treatment could be observed in $378(76.1 \%)$ of all patients. Treatment success was

Pneumologie 2000; 54: 92-96

(c) Georg Thieme Verlag Stuttgart · New York ISSN 0934-8387

\section{B. Menke, D. Sommerwerck, T. Schaberg \\ Pneumologisches Zentrum Unterstedt, Diakoniekrankenhaus Rotenburg/Wümme}

mostly documented as cure $(n=375)$. No documented treatment success was seen in 119 patients (23.9\%). The most important reason for unsuccessful treatment was lost for follow up (transfer out) in $60 / 119$ patients $(51.2 \%)$, followed by death (24/119; $19.8 \%)$, an interrupted treatment (22/199; $18.3 \%)$ and treatment failure $(13 / 119 ; 10.7 \%)$. No documented treatment success was significantly more common in retreatment cases compared to new cases $(p=0.0003)$ and in patients with at least a single drug resistance $(p=0.04)$. Beside these parameters treatment outcome was significantly superior in patients receiving a standard antituberculosis therapy including at least isoniazid and rifampin compared to patients treated with other regimens during both the initial phase $(p=0.0039)$ and the continous phase $(p=0.0021)$ of therapy. Conclusion: In this retrospective study the use of the international definitions for outcome monitoring showed a substantial proportion of patients with unsuccessful therapy. For the evaluation of the success of the tuberculosis programme in Germany a prospective documentation of treatment outcome data in all patients using the international definitions seems essential.

\section{Einleitung}

Die Tuberkulose gilt in den entwickelten Ländern als eine gut kontrollierte Erkrankung. In der Bundesrepublik unterliegt die Tuberkulose der Meldepflicht des Bundes-Seuchengesetzes. Hierdurch ist gewährleistet, dass hinsichtlich der Morbidität und Mortalität ausgezeichnete epidemiologische Daten zur Verfügung stehen. Das Bundesamt für Statistik und das Deutsche Zentralkomitee zur Bekämpfung der Tuberkulose (DZK) werten diese Daten jährlich aus und publizieren sie in geeigneter Form [1]. Das hohe Niveau des öffentlichen Gesundheitsdienstes in der Bundesrepublik sorgt darüber hinaus für eine umfassende Umgebungsuntersuchung, eine Kontrolle der Therapieadhärenz und die weitere Überwachung der Erkrankten nach Beendigung der Behandlung [2]. Allerdings liegen die Daten hinsichtlich der Überwachung nicht in zusammengefasster Form für die Bundesrepublik vor.

Der Therapieerfolg bei Behandlung der Tuberkulose ist unter den Voraussetzungen einer vollen Sensibilität der Erreger gegenüber den Standardmedikamenten, einer guten Compliance und einer intakten Immunität der Erkrankten hoch [3]. In den großen Therapiestudien zur Kurzzeittherapie der Tuberkulose lagen die Heilungsraten am Ende der Therapiephase überwiegend über $95 \%$ und die Rezidivraten nach 3 bzw. 5 Jahren unter $5 \%[4,5,6,7,8,9]$. 
Unklar ist bisher, ob diese in Studien erreichten Erfolgsraten auch in der Praxis der Behandlung in gleichem Ausmaß erreicht werden können. Zur Beantwortung dieser Frage bedürfte es einer standardisierten Erfassung der Langzeittherapieerfolge. Da die Frage des Langzeittherapieerfolges aber für die Beurteilung der Effektivität eines Tuberkulosekontrollprogrammes von essentieller Bedeutung ist, wurden von der Weltgesundheitsorganisation (WHO) in Kooperation mit der International Union Against Tuberculosis and Lung Diseases (IUATLD) und der European Respiratory Society (ERS) Definitionen entwickelt, die eine standardisierte Beurteilung des Therapieerfolges ermöglichen [10,11,12]. Die Anwendung dieser Kriterien erfolgte bisher allerdings in der Bundesrepublik und den meisten europäischen Ländern nicht oder nur partiell.

Wir stellten uns daher die Frage, wie sich die Therapieergebnisse im Einzugsbereich unserer Klinik mindestens drei Jahre nach Beendigung einer von uns initiierten antituberkulösen Chemotherapie darstellten, wenn sie mit den internationalen Kriterien erfasst werden.

\section{Material und Methoden}

Aus den Akten der Klinik identifizierten wir im Jahre 1998 insgesamt 494 Patienten, die zwischen 1980 und 1994 in unserer Klinik mit einer gesicherten Lungentuberkulose (mikroskopischer und/oder kultureller Nachweis von M. tuberculosis) behandelt worden waren. Durch die Wahl dieses Zeitraumes wollten wir sicherstellen, dass einerseits eine genügend große Patientenzahl mit einer vergleichbaren tu-

Tab. 1 Patientendaten

\begin{tabular}{|c|c|}
\hline Geschlecht (Männer/Frauen) & $317 / 177$ \\
\hline Alter (<65 Jahre/ $\geq 65$ Jahre) & $363 / 131$ \\
\hline Herkunft (Deutschland/Ausland) ${ }^{1}$ & $390 / 104$ \\
\hline Unterhalt (erwerbstätig/nichterwerbstätig) ${ }^{2}$ & $401 / 93$ \\
\hline Komorbidität (ja/nein) $)^{3}$ & $239 / 255$ \\
\hline Erkrankungstyp (Ersterkrankung/Rezidiv) & $390 / 104$ \\
\hline Kavernen (ja/nein) & $297 / 197$ \\
\hline Mikroskopie (positiv/negativ) & $408 / 86$ \\
\hline Kultur (positiv/negativ) ${ }^{4}$ & $484 / 10$ \\
\hline Sensibilität (sensibel $/ \geq 1$ Resistenz) ${ }^{5}$ & $311 / 32$ \\
\hline Multiresistenz $\mathrm{H}+\mathrm{R}$ (ja/nein) & 4/339 \\
\hline Therapie Initialphase (Standard/Nicht-Standard) ${ }^{6}$ & $452 / 42$ \\
\hline Therapie Stabilisierungsphase (Standard/Nicht-Standard) 7 & $7430 / 56$ \\
\hline kulturelle Konversion $\leq 8$ Wochen (ja/nein) & $328 / 156$ \\
\hline Mediane Therapiedauer in Monaten (range) & $12(3-30)$ \\
\hline
\end{tabular}

${ }^{1}$ Deutschland: In Deutschland Geborene; Ausland: Nicht in Deutschland Geborene

${ }^{2}$ Erwerbstätig: Erwerbstätige, Rentner, Hausfrauen, Schüler/Studenten; Nicht-erwerbstätig: Arbeitslose, Sozialhilfeempfänger, Asylsuchende

${ }^{3}$ Herz-, Leber-, Nierenerkrankung, Diabetes mellitus, Alkoholkrankheit,

Tumorerkrankung, neurologische Erkrankungen, immunsuppressive Therapie

${ }^{4}$ Kultur negativ: Positive Histologie und/oder Mikroskopie sowie Therapie-

ansprechen

${ }^{5}$ Resistenzen gegen $H, R, Z, E$ und $S$. Nur bei $n=343$ lagen

Sensibilitätsprüfungen vor.

${ }^{6}$ Standardtherapie: $\mathrm{H}+\mathrm{R} \pm \mathrm{E} \pm \mathrm{S} \pm \mathrm{Z}$ für mindestens 8 Wochen

${ }^{7}$ Standardtherapie: $\mathrm{H}+\mathrm{R}$ für mindestens 4 Monate nach Initialtherapie berkulösen Erkrankung zur Verfügung stand, bei der auch das diagnostische und therapeutische Management weitgehend identisch war, und anderseits ein mindestens drei Jahre umfassendes Nachbeobachtungsintervall sichergestellt war. Aus den Unterlagen des Krankenhauses wurden die anthromorphologischen Daten erfasst, sowie Daten zur Herkunft, zum sozialen Status, zur Klinik, zur Diagnostik und zur Therapie der Erkrankung und zu den Begleiterkrankungen (Tab.1). Daten zum weiteren Verlauf der Erkrankung konnten entweder den Unterlagen der Klinikambulanz, den Akten der zuständigen Gesundheitsämter oder den Unterlagen der weiterbehandelnden Ärztinnen und Ärzten entnommen werden. Hierzu wurden ausgedehnte Fragebogen an die jeweiligen externen Institutionen versandt. Der Rücklauf der Fragebogen einschließlich der telefonisch eingeholten Auskünfte war primär vollständig. Konnte allerdings die zunächst angesprochene Institution keine verwertbaren Daten liefern, so erfolgte eine weitere Datenerhebung über die Hausärzte, lokal ansässige Lungenfachärzte, weitere Gesundheitsämter oder Meldebehörden.

Die Klassifikation des Therapieergebnisses wurde nach den tabellarisch dargestellten internationalen Kriterien vorgenommen (Tab. 2). Auf eine Differenzierung der Todesursachen der verstorbenen Patienten musste verzichtet werden, da hierzu für die außerhalb der Klinik Verstorbenen keine verlässlichen Daten erhoben werden konnten.

\section{Tab. 2 Definitionen des Therapie-Ergebnisses}

Erfolgreiche Therapie

Heilung:

Behandlung regelrecht beendet und dokumentierte kulturelle Konversion in der Stabilisierungsphase.

Vollständige Behandlung:

Behandlung durch Ärztin/Arzt regelrecht beendet, jedoch keine dokumentierte kulturelle Konversion in der Stabilisierungsphase.

Nicht-erfolgreiche Therapie

Therapieversagen:

Kulturen blieben positiv oder wurden erneut positiv nach $\geq 5$ Monaten Therapie.

Tod:

Tod an Tuberkulose oder anderen Ursachen vor und während der Therapie.

Behandlung abgebrochen:

Therapieunterbrechung $\geq 2$ Monate.

Ausscheiden der Patienten:

Keine Daten über den Verlauf, Patient unbekannt verzogen, Patient abgeschoben aus Deutschland, keine Dokumentation des Therapieerfolges auffindbar.

Die Auswertung der Daten und die statistischen Berechnungen erfolgten mittels kommerziell erhältlicher Software (JMP for Macintosh, SAS Institute, North Carolina, USA). Die Daten sind als absolute Werte, Prozente oder Mediane mit 95\%Vertrauensbereich angegeben. Der statistische Vergleich der Daten erfolgte mit $2 \times 2$ Kontingenztafeln und dem ChiQuadrat-Test. Die p-Werte wurden mit dem zweiseitigen Fisher-Exact-Test berechnet. Die p-Werte des letzteren wurden als signifikant betrachtet wenn sie $<0,05$ waren. In 
Anbetracht der retrospektiv erhobenen Daten fanden komplexere statistische Analyseverfahren keine Anwendung.

\section{Ergebnisse}

Eine erfolgreiche Therapie konnte bei 76,1\% der Patienten dokumentiert werden. Die mediane Nachbeobachtungsdauer betrug 62 Monate (36-180 Monate). Nahezu ausnahmslos handelte es sich dabei um Heilungen mit kulturell bestätigtem Therapieerfolg (99\%). Unter den Patienten ohne dokumentierten Therapieerfolg (23,9\%) war ein Ausscheiden der Patienten aus der Studie bedingt durch keine weitere Dokumentation des Behandlungsergebnisses nach der stationären Betreuung die bei weitem häufigste Ursache (51,2\%), gefolgt vom Tod (19,8\%), einer abgebrochenen Therapie (18,3\%) und einem Therapieversagen (10,7\%) (Tab.3). Die Patienten ohne dokumentierten Therapieerfolg verteilten sich über den gesamten Untersuchungszeitraum. Ein Cluster hinsichtlich der untersuchten Regionen konnte nicht festgestellt werden.

Tab. 3 Therapieergebnisse

\begin{tabular}{lll}
\hline erfolgreiche Therapie & 378 & $(76,1 \%)$ \\
\hline $\begin{array}{l}\text { Heilung } \\
\text { vollständige Therapie }\end{array}$ & 375 & $(75,5 \%)$ \\
nicht-erfolgreiche Therapie & 3 & $(0,5 \%)$ \\
\hline Therapieversagen & 119 & $(23,9 \%)$ \\
Tod & 13 & $(2,6 \%)$ \\
Behandlung abgebrochen & 24 & $(4,8 \%)$ \\
Ausscheiden der Patienten & 22 & $(4,4 \%)$ \\
\hline \hline
\end{tabular}

Ein signifikanter Einfluss von Patientencharakteristika auf den Therapieerfolg fand sich nur für das männliche Geschlecht $(\mathrm{p}=0,029)$. Die Parameter Alter und Herkunft verfehlten einen signifikanten Einfluss auf den Therapieerfolg nur knapp ( $p=0,069$ bzw. 0,070), wohingegen der Parameter Erwerbstätigkeit ebenso keinerlei Einfluss auf den Therapieerfolg zeigte wie die Tatsache einer weiteren relevanten Komorbidität (Tab. 4).

Tab. 4 Therapieergebnisse und Patientencharakteristika

\begin{tabular}{llll}
\hline Parameter & $\begin{array}{l}\text { erfolgreiche } \\
\text { Therapie }\end{array}$ & $\begin{array}{l}\text { nicht-erfolgreiche } \\
\text { Therapie }\end{array}$ & $\mathrm{P}$ \\
\hline Geschlecht (M/W) & $229 / 144$ & $88 / 33$ & 0,029 \\
Alter (<65/ $\geq 65$ Jahre) & $282 / 91$ & $81 / 40$ & n.s. \\
Herkunft (D/Ausland) & $302 / 71$ & $88 / 33$ & n.s. \\
erwerbstätig/nicht-erwb. & $306 / 67$ & $95 / 26$ & n.s. \\
Komorbidität (ja/nein) & $176 / 197$ & $65 / 56$ & n.s. \\
\hline
\end{tabular}




\section{Diskussion}

In der Bundesrepublik erfolgte über viele Jahre zwar eine Erfassung des Behandlungserfolges bei Patienten mit einer Tuberkulose [1], diese Ergebnisse wurden jedoch nicht regelmäßig regional oder überregional dokumentiert oder veröffentlicht. Auch fanden bei der Erfassung der Daten die internationalen standardisierten Kriterien keine Anwendung. Die von uns erhobenen Daten zeigen, dass eine retrospektive Analyse des Behandlungsergebnisses unter Anwendung der internationalen Kriterien zwar möglich ist, jedoch einen erheblichen Aufwand erfordert und bedingt durch methodische Probleme auch nur tendenzielle Daten liefern kann [13]. Nichtsdestotrotz deutet unsere Untersuchung an, dass die Erfolge der antituberkulösen Chemotherapie in der Praxis mit hoher Wahrscheinlichkeit schlechter sind, als man auf der Basis der Daten aus den großen Therapiestudien erwarten kann.

Auch unter dem Aspekt der eingeschränkten Aussagefähigkeit retrospektiv erhobener Daten erstaunt die Tatsache, dass bei 23,9\% der Patienten keine Aussage über eine definierte Heilung oder vollständige Therapiedurchführung möglich ist. Dabei ist selbstverständlich zu beachten, dass die Gründe für dieses Ergebnis sehr heterogen sind. Einen klaren Grund für eine nicht erfolgreiche Therapie kann man bei den nachgewiesenen Therapieversagern und den Behandlungsabbrechern erkennen. Diese zusammen ca. 7\% der behandelten Patienten ausmachende Gruppe stellt in unseren Augen unter dem Aspekt einer „intention to treat“-Analyse einen vertretbaren und erwartbaren Anteil dar, der sich von den Daten der großen Therapiestudien nicht wesentlich unterscheidet $[14,15,16,17]$. Auch die Tatsache, dass bei $4,8 \%$ der Patienten auf Grund eines vorzeitigen Todes kein dokumentierter Therapieerfolg vorliegt, verwundert nicht, wenn man den hohen Anteil sehr alter (61 Patienten $\geq 75$ Jahre) und polymorbider (239 Patienten mit mindestens einer weiteren wesentlichen Begleiterkrankung) Patienten in unserem Kollektiv betrachtet.

Von deutlich größerem Interesse ist die Tatsache, dass trotz beträchtlichem Aufwand bei 12,1\% der Patienten keine weiteren Daten über den Verlauf der Tuberkulose nach der Entlassung aus der stationären Betreuung erhoben werden konnten. Auf Grund der Struktur der von uns betreuten Population mit einem geringen Anteil an Asylsuchenden oder Flüchtlingen spielt hierbei der Grund Abschiebung keine dominierende Rolle $(n=13 / 60)$. Vielmehr ist der größere Teil dieser Patienten innerhalb der Bundesrepublik Deutschland der Nachbeobachtung und der Dokumentation entgangen $(n=47 / 60)$. Die Gründe hierfür sind vielfältig und mögen zum Teil auch methodisch bedingt sein. Wir denken aber auch, dass die Tatsache, dass ein standardisiertes Erfassungs- und Dokumentationsverfahren des Therapieergebnisses in Deutschland bisher nicht flächendeckend angewendet wurde, einen wichtigen Einfluss auf das hier dokumentierte Therapieergebnis hat. Dabei liegt der Vorteil der standardisierten prospektiven Erfassung des Therapieerfolges in unseren Augen vor allem in der kontinuierlichen Problematisierung der Nachbeobachtung, was schließlich zu einer erhöhten Aufmerksamkeit für die Dokumentation des Therapieerfolges führen kann.
Man kann darüber spekulieren, ob bei einer prospektiven standardisierten Erfassung des Therapieerfolges mittels der WHO/IUATLD-Kriterien die Rate der ausgeschiedenen Patienten kleiner sein würde. Hierfür spricht ein Vergleich mit anderen europäischen Ländern, bei denen eine prospektive Datenerfassung erfolgt. Zwar zeigen die Daten zum Therapieerfolg, die wir in dieser Untersuchung erhoben haben, summarisch keine wesentlichen Unterschiede zu den Daten, die aus den Niederlanden und Italien publiziert worden sind (erfolgreiche Therapie: Italien $=83 \%$, Niederlande $=82 \%$ ) $[18,19]$. Deutlich höher war aber in unserer Untersuchung der Anteil der Patienten, die wegen nicht dokumentierter Daten ausgeschieden sind (Italien: $=4 \%$, Niederlande: $=3 \%$, Niedersachsen $=12 \%$ ).

Ein Vergleich unserer Daten mit den Daten des DZK aus dem Jahre 1997, wo der Therapieerfolg bei 1900 Patienten allerdings nur nach einem Beobachtungszeitraum von einem Jahr überprüft wurde (erfolgreiche Therapie: 74,9\%; 6,1\% ausgeschiedene Patienten) (persönliche Mitteilung M. Forssbohm Wiesbaden 1999) zeigt, dass bei einer prospektiven Anwendung des „Outcome-Monitoring“ hinsichtlich der ausgeschiedenen Patienten bessere Ergebnisse erreicht werden können.

Wenn auch die qualitative Analyse unserer Daten nur mit großer Zurückhaltung erfolgen kann, so zeigen sich hinsichtlich möglicher Einflussgrößen auf den Therapieerfolg mit den Parametern Erst- versus Rezidiv-Erkrankung und Standardtherapie versus Nicht-Standardtherapie doch ein Bild, dass mit der klinischen Erfahrung gut kompatibel ist und wenig überrascht. Es entspricht der Erfahrung, dass Rezidiv-Erkrankungen schlechtere Therapieergebnisse haben [20] und der Erkenntnis, dass die Standardtherapie anderen Medikamentenkombinationen deutlich überlegen ist [21]. Während sich hinter dem Faktor Rezidiverkrankung mit Wahrscheinlichkeit ein erheblicher Anteil von Patienten mit schlechter Compliance verbirgt, spiegeln die Unterschiede hinsichtlich der Therapieregime vermutlich eine ganze Gruppe von Problemen wider (Komorbidität, Unverträglichkeiten, Resistenzen u.a.).

Zusammengefasst zeigen die Daten dieser Untersuchung, dass bei der retrospektiven Erfassung des Therapieergebnisses deutlich schlechtere Resultate gewonnen werden, als theoretisch erwartbar wären. Eine Überprüfung dieser Situation durch die prospektive Anwendung der internationalen Definitionen zum Therapieergebnis bei der Tuberkulose erscheint daher mehr als wünschenswert. Sie würde die Situation in der Bundesrepublik transparenter machen, eventuelle Defizite in der Betreuung der Patienten aufdecken und den Therapieerfolg in Deutschland international vergleichbar machen. 


\section{Literatur}

${ }^{1}$ Deutsches Zentralkomitee zur Bekämpfung der Tuberkulose. 23. Informationsbericht. Berlin: pmi Verlag GmbH 1997

${ }^{2}$ Deutsches Zentralkomitee zur Bekämpfung der Tuberkulose. Empfehlungen zur Infektionsverhütung bei Tuberkulose. Mainz: pmi Verlag GmbH, 1996

${ }^{3}$ World Health Organization and Global Tuberculosis Programme. Global Tuberculosis Control. WHO Report 1998. Geneva: WHO/ TB/98.237, 1998

${ }^{4}$ British Thoracic Society. A controlled trial of 6 -month chemotherapy in pulmonary tuberculosis. Final report: results during the 36 month after the end of chemotherapy and beyond. $\mathrm{Br} \mathrm{J}$ Dis Chest 1984; 78: $330-336$

${ }^{5}$ China Tuberculosis Control Collaboration. Results of directly observed short-course chemotherapy in 112842 Chinese patients with smear-positive tuberculosis. Lancet 1996; 347: 358 362

${ }^{6}$ Hong Kong Chest Service, Tuberculosis Research Centre, Madras, British Medical Research Council. A controlled clinical comparison of 6 and 8 month of antituberculosis chemotherapy in the treatment of patients with silicotuberculosis in Hong Kong. Am Rev Respir Dis 1991; 143: 262 - 267

${ }^{7}$ Hong Kong Chest Service, British Medical Research Council. Controlled trial of 2,4 , and 6 month of pyrazinamide in 6month, three-times-weekly regimens for smear-positive pulmonary tuberculosis, including an assessment of a combined preparation of isoniazid, rifampin, and pyrazinamide. Am Rev Respir Dis 1991; 143: 700 - 706

${ }^{8}$ Singapore Tuberculosis Service, British Medical Research Council. Assessment of a daily combined preparation of isoniazid, rifampin, and pyrazinamide in a controlled trial of three 6month regimens for smear-positive pulmonary tuberculosis. Am Rev Respir Dis 1991; 143: 707 - 712

${ }^{9}$ Singapore Tuberculosis Service, British Medical Research Council. Long-term follow-up of a clinical trial of six-month and fourmonth regimens of chemotherapy in the treatment of pulmonary tuberculosis. Am Rev Respir Dis 1986; 133: 779 - 783

${ }^{10}$ World Health Organization. Tuberculosis programme: framework for effective tuberculosis control. Geneva: World Health Organization, 1994

${ }^{11}$ Veen J, Raviglione M, Rieder HL, Migliori GB, Graf P, Grzemska M, Zalesky R. Standardised tuberculosis treatment outcome monitoring in Europe. Eur Respir J 1998; 12: 505 - 510

${ }^{12}$ Global Tuberculosis Programme. Treatment of tuberculosis: guidelines for national programmes; 2nd ed. Geneva: World Health Organization, 1997

${ }^{13}$ Clancy L, Rieder HL, Enarson DA, Spinaci S. Tuberculosis elimination in the countries of Europe and other industrialized countries. Eur Respir J 1991; 4: 1288 - 1295

${ }^{14}$ Mitchison DA. Treatment of tuberculosis. J R Coll Phys 1980; 14: $91-99$

15 Mitchison DA. Assessment of new sterilizing drugs for treating pulmonary tuberculosis by culture at 2 month. Am Rev Respir Dis 1993; 147: 1062 - 1063

${ }^{16}$ Deutsches Zentralkomitee zur Bekämpfung der Tuberkulose. Richtlinien zur Chemotherapie der Tuberkulose. Pneumologie 1995; 49: $217-225$

${ }^{17}$ Joint Committee of the British Thoracic Society. Chemotherapy and management of tuberculosis in the United Kingdom: recommendations 1998. Thorax 1998; 53: 536 - 548

${ }^{18}$ Veen J, Kalisvaart NA. Index Tuberculosis KNCV. The Hague, 1995
${ }^{19}$ Migliori GB, Ambrosetti M, Besozzi G, Casali L, Raviglione ML. Prospective multicentre study on the evaluation of antituberculosis treatment results in Italy: comparison of the cultureversus smear-based methods. Eur Respir J 1999; 13: 900 - 903

${ }^{20}$ American Thoracic Society. Treatment of tuberculosis and tuberculosis infection in adults and children. Am J Respir Crit Care Med 1994; 149: 1359 - 1374

${ }^{21}$ Mitchison DA. The action of antituberculosis drugs during shortcourse chemotherapy. Tubercle 1985; 66: 219 - 225

\section{Prof. Dr. Tom Schaberg}

Pneumologisches Zentrum Unterstedt Diakoniekrankenhaus Rotenburg/Wümme Verdener Str. 200

D-27356 Rotenburg

E-mail: drschaberg@aol.com 